\title{
GENE REGULATION
}

\section{DISSECTING POLYSOMES}

An improvement on the traditional method of polysome profiling, ribosome density mapping (RDM) can provide information about the distribution of ribosomes on individual mRNAs.

One of the largest remaining challenges in biology is understanding gene expression. Whereas a lot of information is available about eukaryotic gene expression at the level of transcription, as Daniel Herschlag of Stanford University framed the problem, "We know very little about the coordination of what happens after transcription: splicing, RNA export, translation, RNA decay and localization."

To begin a global study of post-transcriptional regulation, Yoav Arava, a postdoc working with Herschlag and Patrick Brown's group at Stanford University, carried out an analysis of translation in yeast using polysome profiling (Arava et al., 2003). Although this study yielded information about how many ribosomes were associated with each mRNA, the data gave no information about the amount of protein produced. But according to Herschlag, "If we know more about the individual steps in translation, we may get some insights into that connection."

To this end, they developed ribosome density mapping (RDM), a method that can be used to determine how many ribosomes are bound to a specific region of an mRNA (Arava et al., 2005). This is a major an advance over polysome profiling, which can yield information only about the total number of ribosomes bound to an mRNA. In both techniques, one lyses cells in the presence of cycloheximide to stop translation and fix the ribosomes on the mRNAs. Then one separates this Iysate by velocity sedimentation in a sucrose gradient, in which mRNAs migrate based on the number of bound ribosomes. Target mRNAs can then be identified by northern blot.

The advance of RDM is that a target mRNA in an individual fraction is cleaved by RNase $\mathrm{H}$ after annealing to an antisense oligodeoxynucleotide complementary to the target mRNA at the desired site of cleavage. When one separates just this treated fraction on a sucrose gradient, the uncleaved polysomes and the two cleaved fragments will have different mobilities corresponding to the number of ribosomes bound. By analyzing different cleavage products, researchers can determine the distribution of ribosomes on the target mRNA.

This method allowed Arava, who has since joined the faculty at the Technion Institute, to address a puzzling result from the global analysis of translation: an unexpected inverse correlation between ribosome density and mRNA length. There were three possible explanations for this observation: limited processivity, inefficient termination, or slower initiation for longer mRNAs. RDM revealed that $5^{\prime}$ and $3^{\prime}$ ends of mRNAs contained similar amounts of ribosomes, suggesting that elongation is processive, and no queue of ribosomes was observed at the $3^{\prime}$ end, indicating that termination is not limiting. Thus, the first two models were eliminated by RDM analysis, leaving the third model, which is consistent with RDM data, as a possibility.

Hershlag pointed out, however, that this is only a small step toward understanding the complex process of translation, and continued: "One of the things we need is more direct in vivo probes of steps in translation as well, which this work does not address, because we are still taking a leap of faith when we compare what is happening in our polysome profiles and what is really going on in the cell."

\section{Irene Kaganman}

\section{RESEARCH PAPERS}

Arava, Y. et al. Genome-wide analysis of mRNA translation profiles in Saccharomyces cerevisiae. Proc. Natl. Acad. Sci. USA 100, 3889-3894 (2003).

Arava, Y., Boas, F.E., Brown, P.O. \& Herschlag, D. Dissecting eukaryotic translation and its control by ribosome density mapping. Nucleic Acids Res. 33, 2421-2432 (2005). 